\title{
La construcción de la identidad de lo femenino: pragmatismo, imaginario $y$ simbolismo
}

\section{DINA MARIA MARTINS FERREIRA}

UNIVERSIDAD MACKENZIE

RESUMEN: En este estudio analizaremos el proceso de designación como organizador del constructo identitario de lo femenino. Esta temática será discutida, en primer lugar, desde el punto de vista de la semántica filosófica, la cual entiende que la relación entre referencia y designación se da en un momento histórico y, por consiguiente, está impregnada de los atributos ideológicos de este momento. Los atributos, a su vez, forman un inventario de los objetivos e intereses de quienes los emplean. En segundo lugar, se indagará si por/en el acto de habla la designación refleja o no un sistema simbólico como una forma cultural especificada en la propia práctica, y si el uso de la lengua está sujeto a formulaciones designativas específicas del imaginario cultural.

PALABRAS CLAVE: designación, referencia, simbólico, imaginario cultural

RESUMO: Nesse estudo analisaremos o processo de designação como organizador do construto identitário do feminino. Vai-se discutir essa temática, primeiramente sob o ângulo de uma semântica filosófica, na qual a relação entre referência e designaçáo é provida de valores ideológicos do momento histórico em que se insere, e, por conseguinte, está impregnada dos atributos ideológicos desse momento. Os atributos, por sua vez, formam um inventário dos objetivos e interesses de que os empregam. Em segundo lugar, se indaga se por e no ato de fala a designação reflete ou não um sistema simbólico como uma forma cultural específica em sua própria prática, e se o uso da língua não está sujeito a formulaçôes designativas específicas da cultura.

PALAVRAS-CHAVE: designação, referência, simbólico, imaginário cultural 
ABSTRACT. In this study we shall analyse the process of denotation as an organizer of the identity construct of the feminine. This problem is approached, in the first place, from the point of view of philosophical semantics, which understands the relation between reference and denotation as situated in a historical moment and consequently pregnant with the ideological attributes of that particular moment. The attributes, in turn, provide an inventory of the objectives and interest of those who use them. In the second place, we shall find out whether by/in the act of speech denotation reflects or not a symbolic system as a specific cultural form in its own practice, and whether the use of language is subject to denotative formulation of specific cultural imaginaries.

KEY WORDS: denotation, reference, symbolic, cultural imaginary

\section{Introducción}

En este estudio analizaremos cómo la designación se vuelve preponderante en la manifestación y construcción de la identidad de lo femenino. Esta temática será discutida, en primer lugar, desde el punto de vista de la semántica filosófica, la cual entiende que la relación entre referencia y designación se da en un momento histórico y, por consiguiente, está impregnada de los atributos ideológicos de este momento. En segundo lugar, se indagará si por/en el acto de habla la designación refleja o no, de algún modo, el producto de un sistema simbólico, si designación es mediación de una forma cultural especificada en la propia práctica, y si el uso de la lengua está sujeto a formulaciones designativas específicas de la cultura. Así, la multivocidad de usos designativos en la lengua presenta una tendencia a la univocidad cultural de símbolos.

Frente a la indicación de múltiples sendas teóricas en torno al eje de designación, el lector, a primera vista, podría tener expectativas en cuanto a desarrollos teóricos sobre el proceso designativo. La posición teórica del estudioso es justamente la contraria: son los datos los que dirigen el proceso, son éstos los que recogen presupuestos teóricos que instrumentalizan la discusión sobre el constructo identitario de lo femenino. Esos datos se recogen del discurso mediático, a partir de la lectura cotidiana de una estudiosa del lenguaje.

La muestra está constituida por los siguientes textos: Por una república mejor, de Carlos Heitor Cony, del diario Folha de São Paulo del 6 de junio de 2002; La dueña del Palacio de la Alvorada, de Tina Vieira, revista Época del 3 de febrero de 2003; y Compañera Marisa de Antônia Márcia Vale, revista Isto $E$, del 8 de enero de 2003. El corpus está basado en el discurso mediático por tratarse de un lenguaje que refleja representaciones socioideológicas de una cultura dada. La elección de estos tres textos se debe, primero, al hecho de que figurativizan a la mujer en el 
desempeño político, durante y después de las elecciones, período muy representativo en el proceso político-cultural de Brasil; segundo, al hecho de mostrar la figura femenina en el espacio político, espacio éste en el que las mujeres están todavía bajo las luces de los informativos debido a que son minoría en un territorio primordialmente masculino.

\section{Hacia una semántica de la designación}

Pensar en una manifestación identitaria en el lenguaje nos lleva a la tríada de la propuesta del "giro lingüístico", en la cual la relación entre sujeto y mundo está intermediada por el lenguaje. Es por la designación en el lenguaje que se tiene acceso al mundo. Para describir mejor la tríada podemos atribuirle funciones: el querer decir estaría relacionado con el sujeto, el decir con el lenguaje y lo dicho con el mundo; para ampliar el juego de palabras y de conceptos, diríamos que el mundo es dicho por el decir, que a su vez es accionado por un querer decir. En este proceso, tal como lo asumimos en nuestra propuesta de trabajo, el mundo habla por lo identitario, el lenguaje se materializa en lengua y el sujeto se revela en lo femenino. Y para especificar aún más el campo del lenguaje, diremos que éste se manifiesta en la lengua, y allí el foco analítico es la especificidad lexical de la designación.

En el texto de Cony, Por una república mejor, la designación sobre la mujer radica en el atributo de la belleza y la del hombre en el de la fealdad, ambos en juegos de poder, éste en el nivel político y aquélla en el visual, respectivamente. $\mathrm{Y}$ en los textos de Antonia Márcia Vale, Compañera Marisa, y de Tina Vieira, La dueña del Palacio de la Alvorada, la figura de la esposa del entonces flamante Presidente Lula se configura por los atributos de discreción de primera dama, de ama de casa que asume tranquilamente sus funciones, y de compañera comprometida.

Entre las distintas conceptuaciones de referencia, nos centramos en la que define referencia como "el acto de referir a una entidad extralingüística o estado de cosas, usando una expresión referente" (Rajagopalan, 2004: 396); se observa que la definición apunta a la presencia de un sujeto que practica un acto de relación con el mundo a través de la designación, en fin, a una visión subjetiva en el acto de referir por la designación. Así, la designación se sumerge en valores subjetivos de carácter sociocultural.

Grandes teóricos de la semántica filosófica orientan nuestra argumentación sobre el proceso designativo. En la metáfora del telescopio, Frege presenta el bedeutung como el lente por el cual el sujeto "ve" y "lee" el mundo; el sentido (zinn) necesita el referente (bedeutung) (Frege, 1980, apud Rajagopalan, 2004) para conectarse al mundo. Por lo tanto, el 
lenguaje, en su naturaleza designativa, es el contacto de significación para/con el mundo. Vale también el razonamiento de que hay varios tipos de expresiones referentes: la que trata de nombres propios y cargos específicos -la descripción singular-, y otra que trata de expresiones generales. Tomamos como ejemplos términos del texto La dueña del Palacio de la Alvorada: (a) Marisa Letícia da Silva y primera dama resultarían ser expresiones singulares y (b) "ama de casa" (administración de la casa) una expresión genérica. Sin embargo, Bertrand Russell (1978) afirma que sólo "nombres lógicamente propios" retienen la naturaleza de singularidad y que por tal naturaleza son innominables; su existencia formaría parte de un posible lenguaje adámico. La descripción singular en realidad tiene referente funcional, atributivo, que se construye por el predicado; por tanto las designaciones serían descripciones funcionales del objeto y no el objeto en sí: primera dama, discreción, ama de casa y otros atributos no serían Marisa en sí, sino atributos que identifican al yo-Marisa. Para completar nuestro propósito analítico, Peter F. Strawson (1980) sostiene que las expresiones hacen referencia en los usos en que están insertas, usos que se perfilan en los contextos históricos en los que se ubican.

Este razonamiento nos lleva a la tesis de la representación, en la cual el lenguaje correspondería a re- y la cosa en sí a la presentación. Así, la presentación se hace por la representación. La presentación sería casi una revelación epifánica que necesita la re-presentación para viabilizar su aparición material, que sería como un proceso mimético de aproximación al original.

En la medida en que el sujeto forma parte del acto de referir en el lenguaje, el acto de referir se constituye de una presentación política, representada en el uso que se hace del lenguaje, uso repleto de creencias políticas que reflejan lo cultural.

\section{Utilitarismo político en el proceso designativo}

Cuando se piensa en proceso designativo, se está subordinado a la idea de que designar a Marisa como una mujer satisfecha con su universo doméstico no significa llegar a la identidad de ese sujeto, sino percibir que al sujeto se le atribuyen papeles por intereses políticos: valorar, tal vez, la simplicidad de una mujer de origen humilde, que llegó a la cima, ocupando el lugar de primera dama de un país. En el texto La dueña del Palacio de la Alvorada, al afirmar que Marisa es "Espontánea entre amigos, a la primera dama le gusta ser quien es - y eso encanta a quienes la conocen", no se está nombrando la personalidad de Marisa, sino asignando un atributo político a los intereses del momento histórico: decir que la 
primera dama es sencilla, una mujer que está feliz con la simplicidad de las tareas cotidianas: "Por ahora se ocupa del asunto que realmente le gusta: la administración de la casa." Esa espontaneidad la ratifica la presencia designativa de la ausencia de un atributo opuesto, la mujer feminista. La alteridad de propiedades, constituida por la categoría del feminismo, enaltece la feminidad tradicional de la ama de casa como complemento de lo masculino:

\begin{abstract}
"Ahora también es la primera dama quien cuida personalmente del día a día en el Palacio de la Alvorada. En un gobierno que abriga el mayor índice de feministas per capita incluso en los ministerios, nadie critica a Marisa. 'Si ella fuera militante, sería mejor', dice Sylvia Camuça, del grupo Articulación de las Mujeres Brasileñas. 'Pero no tenemos esa expectativa.' El presidente, que se divertía con el discurso feminista cuando era dirigente sindical, aprecia el comportamiento de Marisa, mujer que se mostró presente en momentos dramáticos de su vida política."
\end{abstract}

El mismo pragmatismo atraviesa la designación propuesta por Cony, en el texto Por una república mejor, sobre la mujer brasileña, la mujer bella, cuya belleza garantiza votos políticos:

"Dicen que Cleopatra era bonita, hechizó a dos generales romanos, pero se suicidó
con una picada de serpiente. Dicen también que no era gran cosa, tenía la nariz en
forma de gancho como la de un águila. Y en aquel entonces pese a la excelencia de la
medicina egipcia y de sus mandingas milagrosas, los cirujanos como Ivo Pitanguy no
crecían como mala hierba. La reina Victoria era fea, casi enana y Catalina, la Grande,
tampoco era gran cosa. El trono no embellece necesariamente a nadie, aunque el
poder tiene algo de excitante. Entre ir a la cama con una Demi Moore plebeya o con
la reina madre de la Transilvania creo que me resultaría muy fácil decidir. Toco el
tema porque, de repente, después del meteórico y deslumbrante paso de Roseana
Sarney por la contienda presidencial, la clase política, tradicionalmente formada por
hombres feos, algunos hasta horripilantes, descubrió que mujer bonita puede rendir
votos."

El autor hace el juego designativo entre las expresiones belleza, fealdad y poder, en la medida en que las categorías belleza y fealdad acaparan la idea de poder, el poder feo y mal usado por los hombres en el ejercicio político, que necesita la belleza física femenina para adquirir comportamiento positivo: "Ya es tiempo de que mejoremos el visual republicano. Seria un punto de partida para que mejoráramos nuestra propia República." El pragmatismo está en la denuncia contra la fealdad performativa de los políticos masculinos. A pesar del carácter positivo dado a los atributos femeninos, se revelan valores ideológicos según los cuales las mujeres aparecen en el escenario político brasileño únicamente por la capacidad de seducción de su belleza física. El encanto de la apariencia femenina se reduce al plano sensorial, pues en la práctica política su presencia es meteórica. En aquel instante, el constructo identitario femenino se establece como instrumento de crítica política 
contra lo masculino que, bajo una ideología patriarcal, configura la noperformatividad política de lo femenino, al enaltecer su poder físico:

"La elección de Rita Camata para vice de Serra tuvo esa finalidad. Y es posible que los demás candidatos presidenciales estén buscando una compañera con una apariencia que alcance para compensar la fealdad estructural de los cabezas de fórmula. Sugiero un repechaje en un terreno fértil de mujeres buenísimas que agradan a la plebe. Las madrinas de las baterías de las escuelas de samba del carnaval brasileño están ahí, serían excelentes reservas. Nada tengo contra ellas y, sinceramente, me gustaría ver a Luíza Brunet, Luna de Oliveira, Vera Fischer, Adriane Galisteu e incluso a la veterana Dercy Gonçalves como opciones de poder en el caso de emergencia constitucional."

\section{Imaginario-cultural en la designación}

Los objetos nombrados que apuntan hacia allá-afuera, indicativos de figuras femeninas, son los nombres funcionales dados al objeto mujer. A cada expresión sobre la mujer -ya sea la ama de casa sencilla que hace lo que le gusta, ya sea la mujer bonita que genera votos- se modifican funciones que son cambiadas/sustituidas en el predicado identitario de la mujer; el conjunto de variables atributivas se rellena a cada momento del acto designativo. Pero el problema va más allá del utilitarismo del aquíahora, cuando se propone pensar cuál es el vínculo entre el individuo -Marisa, Roseana Sarney, Rita Camata- y su representación social, y cómo éste se esboza en lo cultural. Nos parece que las designaciones que se manejan en la identificación de los individuos "nos pueden brindar pistas preciosas y bien actuales sobre las relaciones sociales y culturales que se establecen entre hombres y mujeres y los significados que se le atribuyen" (Martins, inédito).

El lente que capta la referencia muestra el mundo identitario del instante designativo, pero la nominación puede también configurar una re-actualización del pasado, de lo imaginario, de una historia cultural.

Se advierte el continuo choque entre las fronteras de lo individual con lo social. De un lado el instante performativo del utilitarismo con su heterogeneidad manifiesta, del otro la presencia de lo imaginario que refleja la homogeneidad de lo cultural. Uno no excluye al otro, pues el movimiento de estos dos niveles es de sobreposición y no de causalidad. La paradoja se plantea por la convivencia de la durabilidad de una dimensión que parece homogénea y que se alimenta de lo performativo. Los atributos designativos, en cuanto performativos, de alguna forma tienen su bedeutung "ampliado" en la historia del objeto referido. El nombre del objeto, por la "idea" del objeto, reconoce lo cultural (colectivo) alimentado por lo imaginario (individual inserto en lo colectivo) del sujeto situado en su historia. 
El imaginario dimensiona los modos de hacer del sujeto, ya que imaginario es un parámetro cultural. El imaginario lo mide la colectividad cultural, es un espejo de la cultura, es una construcción histórica y en la medida en que imaginario es pulsión del ser humano, y que sujeto y mundo se intercambian vía lenguaje, el imaginario circula en el lenguaje. Los otros del mundo se hacen presentes en la dimensión imagética cultural que habita en cada sujeto, dimensión que ofrece "rutas" identitarias, es decir, los modelos que deben seguirse para constituir un yo identitario. El imaginario proyecta un modelo que atiende a los intereses del juego sociohistórico; es una búsqueda de un yo estabilizado visto por el otro. En la búsqueda de una homogeneidad el sujeto se estabiliza en la diversidad de lo vivido, lo que muestra que la unidad pertenece a la dimensión de lo imaginario, la estabilidad está provista en el universo imaginario; es una forma, tal vez, de sedimentar e impedir la escisión del multiculturalismo.

\section{Praxis y simbolismo, una convivencia paradójica}

El proceso designativo, además de ser regido por lo imaginario, revela el símbolo por detrás del interés de las prácticas de la vida cotidiana. Pensar en los símbolos de la figura femenina contenida en la propiedad belleza y en la función ama de casa y compañera tiende a borrar las contradicciones, las luchas de poder y los intereses materiales. La creación y en este caso la re-creación simbólica de "Amelia"l demuestra las imposiciones de la esfera sociopolítica; el cambio está en la designación de lo ya vivido, lo pasado es re-presentado, presentado nuevamente. El texto Compañera Marisa ilustra el tema de lo simbólico:

"Durante la campańa, ella estuvo siempre al lado del marido, que a su vez no le ahorró elogios y gestos públicos de cariño como nunca se había visto antes en la vida pública brasileña. La pareja Marisa Letícia y Luiz Inácio Lula da Silva trajo al Planalto un comportamiento que fue símbolo de la revolución en las relaciones entre hombres y mujeres en las décadas de los 60 y 70 : el compañerismo. Lula consulta a Marisa al tomar las decisiones más difíciles."

Es lo que Sahlins (2003: 63) nos indica a partir de la antropología y disciplinas análogas, que la simbología nos da "una versión del pasado como realmente lo vive un segmento de la sociedad, como el mapa de su condición presente." Es el símbolo del compañerismo femenino, que si queremos remonta al mundo de Penélope, siempre a la espera de su marido Odiseo.

En el mundo simbólico, "el ojo que ve es el órgano de la tradición" (Sahlins, 2003: 70), tradición entendida como un conjunto de significa- 
dos acumulados, vividos en la historia que hace de una percepción designativa una concepción que adviene de la praxis social en su especificidad histórica.

La propuesta aquí no es desenmascarar el interés político por detrás del símbolo, sino revelar el símbolo que está por detrás del utilitarismo. Al mostrar a la primera dama como la compañera comprometida con los intereses nacionales, vestida de rojo en homenaje al Sindicato de los Trabajadores, con adornos y ropas de marcas nacionales, la identidad de Marisa, al servicio del interés, también se ajusta al mundo de lo imaginario simbólico, de la mujer que suma, que completa, de la mujer adámica que nace de la costilla del hombre. Por tanto, prácticas sociales, prácticas designativas que advienen de la praxis social revelan símbolos en la vida cotidiana:

"Doña Marisa ha concluido que es hoy una de las mujeres más observadas del país y lo que use será imitado. La industria de la moda es la segunda mayor empleadora del país, sólo pierde frente a la construcción civil y al usar y divulgar los productos nacionales, ella quiere colaborar para que su compañero realice la 'obsesión' de generar nuevos empleos para los brasileños. En el color de la ropa homenajeó al Partido de los Trabajadores, en la elección de los materiales y creadores de los trajes inauguró el 'estilo Marisa' usando productos exclusivamente nacionales de excelente calidad, pero sin hacer ostentación". El vestido fue diseñado y confeccionado en muselina brasileña por el estilista Walter Rodrigues. Los zapatos y la cartera son de la marca Ferri, de São Paulo."

Nos parece que el conflicto entre utilitarismo y enfoque cultural no debe ser medido por un condicionamiento ilimitado entre las dos dimensiones, incluso porque el conflicto se disipa en la relación que se establece entre las "partes", es decir,

"si el orden cultural hay que concebirlo como la codificación de la acción intencional y pragmática real del hombre, o si, al contrario, la acción humana en el mundo debe ser comprendida como mediada por el proyecto cultural, que ordena inmediatamente la experiencia práctica, la práctica ordinaria y la relación entre ambas" (Sahlins, 2003: 61).

La semejanza de rasgos conceptuales que perfila la unicidad cultural, se constituye de las "diferencias en significados y usos determinados localmente" (Sahlins, 2003: 73). No hay fronteras delimitadas entre el universo cultural, la práctica social y el performativo individual, incluso porque uno no deriva automáticamente del otro. La cultura estandariza rasgos por la especificidad de lo particular; como totalidad, se rige por ideas dominantes, que se vuelven dominantes porque surgen de la diferencia de lo que es no-dominante. Si diéramos a la cultura un carácter unívoco de significación estaríamos más cercanos a una clasificación reductora de identidades que a una representación sociohistórica. La 
historia es un constructo, es el resultado de performativos transformadores.

Lo que se nota es que ninguna de las áreas excluye a la otra, pues hay un continuo movimiento anatómico de performativos sociales que modelan la cultura y lo imaginario como parte del proceso designativo y que a su vez se manifiestan en el uso de la lengua. Esta relación no puede ser otra cosa que una superposición. Sin lugar a dudas la praxis social y el lenguaje están sujetos a las formulaciones designativas de la cultura y viceversa. Debe quedar claro que la "orientación del efecto lógico" (Morgan, apud Sahlins, 2003: 16) a partir de las circunstancias que llevan a la práctica y que se organiza y codifica institucionalmente no hace sentido, pues el proceso no es de causalidad lineal, sino de un tipo de convivencia anatómica.

El utilitarismo del uso designativo puede reforzar las significaciones ya cristalizadas en lo sociocultural desvelando la durabilidad de un referente modalizado, que atiende a significaciones simbólicas.

En realidad recorremos un camino metodológico que tuvo como objetivo presentar la representación de lo femenino, sus usos designativos, los cuales se influencian por lo imaginario en el acto de referir, designando en relación con un referente incluso simbólico, que atraviesa la cultura de los sujetos.

Como dice Rajagopalan, el poder de la designación es más grande de lo que se pueda imaginar, pues alimenta tanto cambios como interpretaciones fantasiosas de los mismos:

"los medios imprimen ciertas interpretaciones por el simple acto de designación de determinados sucesos (...) Ya que, de acuerdo con ciertas teorías semánticas de gran prestigio, los nombres no son más que meras etiquetas identificadoras de objetos, hay que pensar más allá de la semántica de los nombres propios para encarar el fenómeno de la nominación como un acto eminentemente político." (Rajagopalan, 2003)

La designación es siempre representación y nunca presentación de la cosa en sí, del ser femenino. El ser femenino está representado por designaciones, designaciones que forjan sentidos y referentes inducidos por el momento utilitario de un sujeto, de un grupo, de una cultura: "la designación es un proceso por el cual nosotros le damos una ubicación local y un nombre a un objeto (...) tal vez el paso más importante hacia la manipulación ideológica." (Rajagopalan, 2003).

En el juego entre sujeto, lenguaje y mundo, podríamos afirmar que la identidad es un constructo de atributos que se alinean a lo largo de la historia, puesto que son designaciones, bajo el punto de vista pragmaticista y/o simbólico, "no son más que representaciones verbales de atributos y los atributos son características accidentales (y no esenciales)" (Rajagopalan, 2003, serial), de un presente o de un pasado re-actualiza- 
do: mundos de referentes que nos atraviesan, que nos construyen y que construimos.

El constructo identitario engendraría en el discurso un efecto Penélope - tejer, destejer, tejer nuevamente en busca de una ilación ideal que atienda no sólo al instante pragmático del acto de designar en una lengua como también a las valoraciones cristalizadas del sistema simbólicocultural en el que se ubica. La elección designativa atribuye valores al objeto nombrado.

\section{NOTA}

1 La etimología de Amelia o Amalia podría explicar la metáfora propuesta al significar una laboriosa abeja o una hormiguita, pues esa mujer-Amelia, al igual que la abeja y la hormiga, trabaja secreta e incansablemente en su vida cotidiana en pro de su familia, sin quejarse. Pero también podemos añadir a la etimología del término la letra de una canción brasileña tradicional, muy conocida, que habla de una mujer llamada Amelia, "la mujer de verdad". En ambos casos Amelia es el símbolo de la feminidad, de la mujer que es compañera del hombre para/en todos los momentos.

\section{REFERENCIAS BIBLIOGRÁFICAS}

FELDMAM-BIANCO, B. y CAPINHA, G. (comps.) (2000) Identidades-Estudos de cultura e poder. São Paulo: Hucitec.

FERREIRA, D. (2002) Discurso feminino e identidade social. São Paulo: FAPESP/ Annablume.

FERREIRA, D. (en prensa) 'Political discourse in the feminine manifestation: social identity (Queen Marianna of Spain and Governor Roseana of Maranhāo)', Image 2002. Denmark: Alborg University.

FERREIRA, D. (2003) Culture and society - the identity of the feminine manifestation, Journal of Language and Linguistics, volumen 2, número 1. Disponible en World Wide Web: <www.jllonline.net>. [Consulta: 24 febrero 2006].

FREGE, G. (1980) Os fundamentos da aritmética. In Os Pensadores, pp. 20-278. Rio de Janeiro: Editora Abril.

MARTINS, E. (inédito) Os nicknames nas salas da Internet: novos nomes, velhos mitos.

RAJAGOPALAN, K. (2003) 'Designação: a arma secreta, porém incrivelmente poderosa, da mídia em conflitos internacionais', GEL XXVIII $50^{\circ}$ Seminário do GEL/2002. São Paulo: Unitau Unitau/USP, Taubaté. CDROM.

RAJAGOPALAN, K. (2004) 'Reference', en P. Strazny (org.) Encyclopedia of Linguistics, No.2, pp. 396-398, Nueva York.

RUSSEL, B. (1978) 'Lógica e conhecimento', en Os Pensadores, pp. 3-315. Rio de Janeiro: Editora Abril.

SAHLINS, M.. (2003) Cultura e razão prática. Rio de Janeiro: Zahar.

STRAWSON, P. F. (1980) 'Escritos lógico-lingüísticos', en Os Pensadores, pp.261345. Rio de Janeiro: Editora Abril. 
ZIZEK, S. (2002).O paradigma das ideologias, Folha de Säo Paulo, Caderno Mais!, p.3.

DINA MARIA MARTINS FERREIRA, es lingüista, docente de la Universidad Presbiteriana Mackenzie, São Paulo, Brasil. Realizó sus estudios de doctorado en la Universidad Federal de Río de Janeiro, Río de Janeiro, Brasil. Su postdoctorado lo realizó en la Universidad Estadual de Campinas, Campinas, São Paulo, Brasil. Es autora de los libros Discurso Feminino e Identidade Social (2002), coedición Annablume/FAPESP, Fundación de Apoyo a la Investigación del Estado de São Paulo, y Não pense, veja - O espetáculo da linguagem no palco do Fome Zero (2006), editorial Annablume. Ha compilado, en colaboración con el Prof. Kanavillil Rajagopalan, el libro "Políticas em Linguagem: perspectivas identitárias" (2006), Editorial Mackenzie. Ha publicado varios artículos en revistas internacionales y nacionales y ha contribuido con capítulos en libros.

Correo electrónico: dinaferreira@terra.com.br

Traducción: Magali de Lourdes Pedro 